\title{
A preliminary study of the oral microbiota in Chinese patients with Sjögren's
}

\section{Syndrome}

Miaoran Lia,b, Yan Zoua,b, Qian Jianga ${ }^{a}$, Lindi Jiang ${ }^{c}$, Qiang $\mathrm{Yu}^{\mathrm{c}}$, Xiaojun Ding ${ }^{\mathrm{a}, *}$, Youcheng $\mathrm{Yu}^{\mathrm{a}}$

a Department of Stomatology, Zhongshan Hospital, Shanghai Medical School, Fudan University, 111 Yixueyuan Road, Shanghai 200032, China;

c Department of Rheumatology, Zhongshan Hospital, Shanghai Medical School, Fudan University, 111 Yixueyuan Road, Shanghai 200032, China.

* Correspondence author, Department of Stomatology, Zhongshan Hospital, 111 Yixueyuan Road, Shanghai 200032, China; Tel: 086-021-64041990-2585; Fax: 086-021-64038472; E-mail: ding.xiaojun@zs-hospital.sh.cn

b These authors contributed equally. 


\section{Abstract}

Objective: To investigate the oral microbiota in Sjögren's syndrome (SS) as opposed to that of healthy subjects.

Study design: Ten patients with primary SS, [6 patients daily taking stable dosage of hydroxychloroquine $(\mathrm{HC})$ and 4 patients taking hydroxychloroquine combined with Prednisone Acetas ( $\mathrm{HC}+\mathrm{PA})]$, along with 10 age-matched healthy controls were examined in regard of number of teeth, stimulated/unstimulated saliva secretion rate. Microflora on bilateral buccal mucosa was analyzed by high throughput sequencing. Statistical analyses were performed using the chi-square test, $t$ test and Mann-Whitney U test. The Venn diagrams and Redundancy Analysis (RDA) were also used to evaluate effects of the disease and treatment on the bacterial community composition.

Results: The relative abundance of Proteobacteria in SS group was lower compared to controls $(P=0.002)$. The total richness of genera for all groups was 339 . The numbers of genera in SS group and in control group were 248 and 270, respectively. Some taxa with different prevalence and/or relative abundance were found between two groups.

Conclusions: SS affects the oral microbiota and SS patients carry a different and less diverse microorganism community compared with healthy subjects. Prednisone Acetas is an influence on the oral microbiome. This study provides a basic data on the oral flora in SS patients.

Keywords Sjögren's syndrome; oral microbiome; Prednisone Acetas 


\section{Introduction}

Sjögren's syndrome (SS) is a systemic chronic autoimmune disease which is characterized by dryness of the mouth and eyes and impairs oral health. Subjective xerostomia has been reported in $75.18 \%$ to $91.84 \%$ patients with SS [1]. As a sequence, notable oral features include increased dental caries especially root caries, oral candidiasis and angular cheilitis. To clarify the inherent mechanism, the change of oral flora in SS patients has been widely investigated since 1974 [2]. The shift of oral microbial composition related to caries, periodontitis and mucosal infection has been discovered basically with culture-based techniques [2-13]. Although most of the researchers attributed the characteristics of oral micro flora in SS patients to reduced salivary flow and associated qualitative changes in saliva and their sequelae, the possible changes of host and pathogens interaction cannot be neglected. The host immune system status alters because of the disease itself, as well as the related medication. To control the disease, some medicines include prednisone and hydroxychloroquine are often prescribed for SS patients. These medicines have the function of altering the host immune reaction to pathogens. However, the influence of medicine used by SS patients on oral flora has been less studied.

To explore the microbiota in oral cavity, culture-dependent approaches were usually adopted. However, there are more than 700 bacterial species have been detected in the oral cavity and over $50 \%$ of oral bacterial species have not yet been cultivated and could not been detected by means of culture [14,15]. Advances in molecular technology have made it possible to detect most of the species, whenever cultivable 
or uncultivable, and to explore the diversity of the microbial community [16-18]. Except terminal restriction fragment length polymorphism (T-RFLP) [3,6], Ribosomal $16 S$ cloning and sequencing is the widely used new technology in research of diversity of microbial community. It has been used to define the healthy "core microbiome" of oral microbial communities and investigate the relationship between dental caries/periodontitis and the diversity of the microbial communities $[17,19,20]$. Even though, ribosomal $16 \mathrm{~S}$ cloning and sequencing has not been used in SS patients and little has been done about the relationship between SS and oral microbiota, especially uncultivable bacteria.

The aim of this preliminary study was to elucidate the relationship between SS and buccal mucosa microbiota and the effects of Prednisone Acetate on the microbiota of SS patients using high throughput sequencing.

\section{Materials and methods}

\section{Subjects}

The study population consisted of 20 non-smoking Chinese female subjects (aged 21

to 80 years), of whom 10 patients were diagnosed as having primary SS (American-European criteria for the classification of Sjögren's syndrome, 2002), and 10 were healthy individuals. SS suffers were recruited in Department of Rheumatology, Zhongshan Hospital, Fudan University. None of them had oral mucosa infection, used saliva-stimulating agents and fluorides, and received periodontal, antibiotic or antimycotic therapy over the last three months. Subjects with 
removable denture and oral implant also were excluded. Daily taking stable dosage of hydroxychloroquine $(\mathrm{HC})(0.2 \mathrm{~g} / \mathrm{d})$ or hydroxychloroquine combined with Prednisone Acetas (PA) $(10 \mathrm{mg} \sim 20 \mathrm{mg} / \mathrm{d})$ for at least 3 months was also necessary for selection criteria. The 10 first subjects fulfilling the criteria were included in the study. Healthy controls were recruited from the family members of employees of Zhongshan Hospital, Fudan University. All controls had the normal salivary secretion rates and were matched according to age, number of teeth, periodontal and mucosal status. The study was approved by the Ethic committee at Zhongshan Hospital, Fudan University. All subjects provided written informed consent before participation in this study.

\section{Clinical examination and sampling}

Each participant accepted the examination and sampling between 9 a.m. and 11 a.m.

Prior to the visit, they were asked not to brush their teeth and not to eat or drink for at least 2 hours before the test. All the clinical tests, the questionnaire survey and sampling were carried out by a single experienced and calibrated dentist. Samples were taken before other tests with sterile cotton swabs on bilateral buccal mucosa. Oral examination comprised the evaluation of the clinical aspects of the oral mucosa, number of teeth and stimulated/unstimulated secretion rate. The unstimulated secretion rate was measured for 15 minutes. The subject leant forward and passively allowed saliva to flow into a test-tube. The stimulated secretion rate was measured using paraffin wax for 5 minutes. Stimulated/unstimulated whole salivary samples were taken and flow rates were calculated according to Almståhl described method [21]. Ocular staining scores were taken from patients' ophthalmology inspection 
records.

\section{DNA isolation and sequencing}

DNA was isolated according to previously described procedures using lysozyme and proteinase $\mathrm{K}$ treatments and a DNeasy blood and tissue kit (Qiagen) [22]. Amplicon libraries of $16 \mathrm{~S}$ rRNA gene V1-V3 hypervariable regions were generated as previously described [23]. In brief, PCR amplification was carried out in two steps. The first step included $2 \quad \mu l \quad(5 \quad p M)$ 533R inner primer: 5'-GAGTTCCTTGGCACCCGAGAATTCCATTACCGCGGCTGCTGGCAC-3' in $22 \mu \mathrm{l}$ PCR system. The samples were run for 8 cycles using the following parameters: $98^{\circ} \mathrm{C}$ for $10 \mathrm{~s}, 50^{\circ} \mathrm{C}$ for $90 \mathrm{~s}$, and $72^{\circ} \mathrm{C}$ for $30 \mathrm{~s}$. The second step was adding other primers $(8 \mathrm{~F}$ inner primer: 5'-TTCCCTACACGACGCTCTTCCGATCTAGAGTTTGATCCTGGCTCAG- $\quad 3{ }^{\prime}, \quad$ 8F outer primer: 5'-AATGATACGGCGACCACCGAGATCTACAC-NNNNNNNN-TCTTTCCCTACACG ACGCTC-3', and 533R primer: 5'-CAAGCAGAAGACGGCATACGAGAT-NNNNNNNN-

GTGACTGGAGTTCCTTGGCACCCGAGA-3') $1 \mu \mathrm{l}(10 \mu \mathrm{M})$ respectively to PCR system. The amplicons were generated in PCR reactions using 30 cycles of $98^{\circ} \mathrm{C}$ for 10 seconds, $50^{\circ} \mathrm{C}$ for 90 seconds, and $72^{\circ} \mathrm{C}$ for $30 \mathrm{~s}$. After purification, the amplified products were sequenced on a NGS illumine Miseq 2 x 300bp platform.

\section{Data analysis}

Sequencing data were trimmed. Primer sequences and low-quality data, sequences 
that had an ambiguous base call in the sequence, or that were shorter than 200bp after trimming were removed. For operational taxonomic unit (OUT)-based analysis, sequences were clustered using mothur and a 3\% dissimilarity cutoff. Every sequence was aligned with silva data set and definition of a sequence's taxonomy was determined using a pseudobootstrap threshold of $80 \%$ [24]

\section{Statistical analysis}

Normality of distribution of qualitative variables was verified by means of one-sample Kolmogorv-Smirnov test. For demographic and clinical data, normally distributed data were compared via $t$ test, otherwise with nonparametric test. Differences in taxon prevalence were determined via chi-square test, while differences in relative abundances of individual genera and phyla were tested via $t$ test or Mann-Whitney $U$ test, depending on the normality status. Only genera with 10 or more sequences were included in prevalence and relative abundance comparisons. The Venn diagrams and Redundancy Analysis (RDA) were made with mothur. RDA was used to evaluate effects of the disease and treatment on the bacterial community composition.

\section{Results}

\section{Demographic and clinical characteristics of subjects included in this study}

Table 1 shows the demographic and clinical information of SS and control subjects. The stimulated/unstimulated secretion rate of saliva in SS group was much lower than the control group. These two groups did not differ in terms of parameters related to oral health. 


\section{The diversity of buccal mucosa bacterial communities in SS patients}

After optimization, a total of 366,452 sequence reads were included in the final analysis. The range of sequence reads obtained per subject varied from 13,470 to 24,236 . A total of 30,418 OTUs were observed across all subjects and the range of OTUs number varied from 1,890 to 3,292 . The range of genera obtained per subject varied from 54 to 168 . A total of 339 genera were identified across all subjects and the number of genera in SS was less than that in control groups $(87 \pm 11$ and $97 \pm 11$, respectively). However, the difference was not statistically significant $(P>0.05)$. A total of 10 bacteria phyla were responsible for $>99 \%$ of all sequence reads in two groups (Fig 1). The relative abundance of Proteobacteria in SS group was lower compared to controls $(P=0.002)$.

The Venn diagrams were used to depict the distribution of buccal mucosa bacterial communities (Fig 2). The total richness of genera for two groups was 339. The numbers of genera in SS group and in control group were 248 and 270, respectively. The number of genera shared between two groups was 179, which accounted for $52.80 \%$.

Difference in prevalence of individual taxa between SS and control groups were then evaluated. One genus (Ralstonia) of Proteobacteria with significantly different prevalence between two groups was detected (Fig 3). Difference in relative abundance of individual taxa between SS and control groups were also evaluated. The 10 genera differentially represented in terms of relative abundance in each group were shown in Fig 4. Genera increased in SS group included Leucobacter, Delftia, 
Pseudochrobactrum, Ralstonia and Mitsuaria, amongst which, the first belongs to Actinobacteria and the last four belong to Proteobacteria. The genus with decreased relative abundance in SS group was Haemophilus, Neisseria, Comamona, Granulicatella and Limnohabitans, amongst which, Granulicatella belongs to Firmicutes and the other four are genera of Proteobacteria, too.

PA influences the diversity of buccal mucosa bacterial communities in SS patients

To investigate the effect of prednisone on oral microbiota, SS group was divided to two subgroups according to whether taking Prednisone Acetas (PA) or not. There were no statistically detected demographic and clinical differences between two subgroups, in words of age, number of teeth and stimulated/unstimulated secretion rate.

Next, to explore the effect of PA on the diversity of the microbiota of buccal mucosa, genera-based redundancy analysis (RDA) was executed. Only 25 genera with the highest relative abundance were included in the analysis. Most of these 25 genera are commensal bacteria and opportunistic pathogens. Controls, $\mathrm{HC}$ and $\mathrm{HC}+\mathrm{PA}$ group were located in different quadrants. Streptococcus and Lactobacillus were among the genera obviously affected by the treatment of PA, while Haemophilus, Neisseria, Streptococcus, Lactobacillus, Capnocyophaga, Corynebacterium, enterobacteria, Leptotrichia and Prevotella, et cetera were among the genera affected by the disease of SS (Fig 5). 


\section{Discussion}

There are 4 major microbial ecosystems in oral cavity, which are the dorsum of the tongue, the smooth mucosa, the supragingival tooth surfaces, and the gingival crevicular region, due to differences in surfaces structure such as availability of nutrients, and oxygen tension [10]. The composition of microbial flora is specific in each of these areas. According to Zaura [17], community of cheek samples shows the least diversity, so this preliminary study investigated the buccal mucosa microbial flora of SS patients.

With traditional culture--dependent approaches, oral microbial samples were studied for some microorganisms associated with caries, gingivitis, periodontitis and mucosal infections [2,4,5,7-13]. Although Leung et al [7] investigated more than 30 species of oral bacteria from SS subjects with culture--dependent approaches; other researchers usually chose only several genera/species of microorganisms for their studies because of the limitations of culture [2,4,5,8-13]. Here, we used high throughput sequencing to investigate buccal mucosa microbiota in SS subjects and studied more than 30,000 OTUs, among which were 339 genera or more than 500 classified species. The results showed that SS patients had the less diversity of oral microbiota than controls had. Based on either phylum level or genus level, we found microbiota differences between SS and control groups. In this study, it was similar to the results form T-RFLP research that Neisseria was with lower relative abundance than in controls [3]. However, increased prevalence and relative abundance of Ralstonia and change of relative abundance of Delftia, Pseudochrobactrum, Leucobacter, Mitsuaria, 
Haemophilus, Comamona, Granulicatella and Limnohabitans in SS have not been found in related researches. Some species of these genera have not been cultured successfully so far.

Ralstonia is ubiquitous environmental organism of low virulence, colonizing inside of mouth and lung of healthy nonsmokers, and do not usually cause illness in immunocompetent hosts $[25,26]$. Interestingly, SS group had increased both prevalence (present in 5/10 SS patients) and relative abundance of Ralstonia, simultaneously. However, the prevalence of Ralstonia was not dependent on HC use (Supplementary Fig 1: phylogenetic tree of $\mathrm{HC}+\mathrm{PA}$; Supplementary Fig 2: phylogenetic tree of $\mathrm{HC}$ ). This indicates that the oral ecosystem of SS patients is suitable for growth of Ralstonia and HC might have no effects on it.

Factors influencing the oral microbiota composition include age, gender, dietary habits, smoking, oral hygiene, use of antibiotics and, probably, genetic factors [16]. Changes of the oral flora in SS are often attributed to hyposalivation which is associated with the lack of clearance function and buffering action of saliva as well as a decrease in antimicrobial salivary components $[3,11,27]$. Hyposalivation can be caused by SS, radiation therapy, and unknown factors [9,21]. Some medications are usually thought as one important reason causes xerostomia/hyposalivation [28,29]. Neuroleptics and cyclic antidepressants, such as desipramine, induce changes in cultivable oral microbiota at least partially through alterations of saliva [30]. Other medications, like treatment with immunosuppressant, also can result in the changes of the oral flora [31-33]. As one of the immunosuppressants, prednisone has been 
found positively correlated with bacterial richness in solid-organ transplant recipients [33]. Similarly, PA affected the oral microbiota in SS patients in our study according to the RDA results. However, it is still unknown these drugs indeed reduce salivary flow and the inherent calibrated mechanisms still wait to be investigated $[28,29]$.

The diversity of oral flora may be related to some complications in some subjects. The oral microbiome is affected in patients who develop respiratory signs and symptoms after allogeneic transplantion [34]. Fricke et al [35] also found that specific differences in the pretransplant oral microbiota were associated with rejection events after renal transplantation. Here, the different microbial flora of buccal mucosa reflected the status of oral disorders in the subjects with SS compared to controls and the relative clinical significance of the change should be clarified in the future.

It is now widely accepted that skin, gut and oral microbiota plays a critical role in the development of the human immune system [36]. Some organisms are even associated with autoimmune diseases. A single gut bacteria Prevotella copri was implicated in the pathogenesis of the rheumatoid arthritis [37]. Unexceptionally, the serum antibody levels to some oral microorganisms have been found elevated or lower in SS subjects compared to healthy controls [27]. Recently, it was demonstrated by Szymula [38] that peptides derived from normal human microbiota can activate Ro60-reactive $\mathrm{T}$ cells. These organisms belong to genera of Prevotella, Capnocytophaga and Corynebacterium. Amongst these, as showed in our results of RDA, genera of Capnocytophaga and Corynebacterium were affected by SS, whether PA taken or not, and genera of Prevotella was affected mostly by SS, especially not 
taking PA. Although we detected the changes of oral flora in SS after medications, combing our results with Szymula's recent findings, it still can provide valuable clues to understanding the possible influence of oral microbiota on autoimmunity. Thus, the change of oral microbiome and immune responses to commensal microbiota and opportunistic pathogens should be explored as potential triggers for initiating autoimmunity in Sjögren's syndrome.

This is a preliminary study and there is no reference supplying related odds ratios for power analysis to calculate the sample size. Even more, number of subjects more than 10 is not suitable to show the diagrams of phylogenetic tree clearly. In some oral microbiome related references (Zaura E et al, BMC Microbiol 2009; Lazarevic V et al, BMC Genomics 2010; Ames NJ et al, PLoS One 2012), only 3, 5 or 11 subjects were included in high throughput sequencing[16,17,34]. Zaura[17] compared the oral microbiome of shedding surfaces and that from solid surfaces in three healthy subjects. Lazarevic[16] study the salivary microbiota from five adults at three time-points to show the inter- and intra-individual variation. To do comparison, Ames[34] divided 11 subjects to two groups (six patients in one group and five patients in the other group). So, although the low numbers, we still divided $10 \mathrm{SS}$ patients into two subgroups depending on corticosteroid use.

There are some limitations of this study. First, the number of subjects is low. Much more patients and controls will be collected in our study to show the difference of prevalence/ abundance of taxa in the future. Second, this is a semi-quantitative study and absolute abundance cannot be obtained. To acquire absolute abundance of each 
taxon, it is necessary to carry out Quantitative Real-time PCR using standard strains as reference. Third, clinical indications of our findings still need to be further explored for prevent and treatment of SS. The oral microbiota should better to be investigated before medication of SS or even in well-designed prospective study.

In conclusion, we examined the buccal mucosa microbiota in Sjögren's syndrome patients using high-throughput technology and found that the microbial community changed in SS and the diversity might be affected by prednisone treatment regardless of cultivability of the bacteria. This study provides a basic data on the oral flora due to SS and gives a hint in etiology of the disease.

\section{Acknowledgments}

This work was granted by the National Science Foundation of China (grant no. 81001202). Kindly technologically assistance was offered by TinyGene Biotechnology Co., Ltd..

\section{Reference}

1. Boutsi EA, Paikos S, Dafni UG, Moutsopoulos HM, Skopouli FN. Dental and periodontal status of Sjögren's syndrome. J Clin Periodontol 2000;27:231-5.

2. MacFarlane TW, Mason DK. Changes in the oral flora in Sjögren's syndrome. J Clin Pathol 1974;27:416-9. 
3. Hayashi Y, Saito T, Ohshima T, Nakagawa Y, Arita T, Yashima A, et al. Terminal RFLP analysis to determine the oral microbiota with hyposalivation. Arch Microbiol 2014;196:489-96.

4. Guobis Ž, Kareivienè V, Basevičienè N, Paipalienè P, Niedzelskienè I, Sabalys G, et al. Microflora of the oral cavity in patients with xerostomia. Medicina (Kaunas) 2011;47:646-51.

5. Ergun S, Cekici A, Topcuoglu N, Migliari DA, Külekçi G, Tanyeri H, et al. Oral status and Candida colonization in patients with Sjögren's Syndrome. Med Oral Patol Oral Cir Bucal 2010;15:e310-5.

6. Almståhl A, Carlén A, Eliasson L, Lingström P. Lactobacillus species in supragingival plaque in subjects with hyposalivation. Arch Oral Biol. 2010;55:255-9.

7. Leung KC, Leung WK, McMillan AS. Supra-gingival microbiota in Sjögren's syndrome. Clin Oral Investig 2007;11:415-23.

8.Eliasson L, Carlén A, Almståhl A, Wikström M, Lingström P. Dental plaque pH and micro-organisms during hyposalivation. J Dent Res 2006;85:334-8.

9. Almståhl A, Wikström M, Stenberg I, Jakobsson A, Fagerberg-Mohlin B. Oral microbiota associated with hyposalivation of different origins. Oral Microbiol Immunol 2003;18:1-8.

10. Almståhl A, Wikström M, Kroneld U. Microflora in oral ecosystems in primary Sjögren's syndrome. J Rheumatol 2001;28:1007-13. 
11. Almståhl A, Wikström M, Groenink J. Lactoferrin, amylase and mucin MUC5B and their relation to the oral microflora in hyposalivation of different origins. Oral Microbiol Immunol 2001;16:345-52.

12. Almståhl A, Kroneld U, Tarkowski A, Wikström M. Oral microbial flora in Sjögren's syndrome. J Rheumatol 1999;26:110-4.

13. MacFarlane TW. The oral ecology of patients with severe Sjögren's syndrome. Microbios 1984;41:99-106.

14. Aas JA, Paster BJ, Stokes LN, Olsen I, Dewhirst FE. Defining the normal bacterial flora of the oral cavity. J Clin Microbiol 2005;43:5721-32.

15. Dewhirst FE, Chen T, Izard J, Paster BJ, Tanner AC, Yu WH, et al. The human oral microbiome. J Bacteriol 2010;192:5002-17.

16. Lazarevic V, Whiteson K, Hernandez D, François P, Schrenzel J. Study of interand intra-individual variations in the salivary microbiota. BMC Genomics 2010;11:523. 17. Zaura E, Keijser BJ, Huse SM, Crielaard W. Defining the healthy "core microbiome" of oral microbial communities. BMC Microbiol 2009;9:259.

18. Segata N, Haake SK, Mannon P, Lemon KP, Waldron L, Gevers D, et al. Composition of the adult digestive tract bacterial microbiome based on seven mouth surfaces, tonsils, throat and stool samples. Genome Biol 2012;13:R42.

19. Aas JA, Griffen AL, Dardis SR, Lee AM, Olsen I, Dewhirst FE, et al. Bacteria of dental caries in primary and permanent teeth in children and young adults. J Clin Microbiol 2008;46:1407-17. 
20. Kumar PS, Leys EJ, Bryk JM, Martinez FJ, Moeschberger ML, Griffen AL. Changes in periodontal health status are associated with bacterial community shifts as assessed by quantitative $16 \mathrm{~S}$ cloning and sequencing. J Clin Microbiol 2006;44:3665-73.

21. Almståhl A, Wikström M. Microflora in oral ecosystems in subjects with hyposalivation due to medicines or of unknown origin. Oral Health Prev Dent 2005;3:67-76.

22.Diaz PI, Dupuy AK, Abusleme L, Reese B, Obergfell C, Choquette L, et al. Using high throughput sequencing to explore the biodiversity in oral bacterial communities. Mol Oral Microbiol 2012;27:182-201.

23. Faith JJ, Guruge JL, Charbonneau M, Subramanian S, Seedorf H, Goodman AL, et al. The long-term stability of the human gut microbiota. Science 2013;341:1237439. 24. Kunin V, Engelbrektson A, Ochman H, Hugenholtz P. Wrinkles in the rare biosphere: pyrosequencing errors can lead to artificial inflation of diversity estimates. Environ Microbiol 2010;12:118-23.

25. Morris A, Beck JM, Schloss PD, Campbell TB, Crothers K, Curtis JL, et al. Comparison of the respiratory microbiome in healthy nonsmokers and smokers. Am J Respir Crit Care Med 2013;187:1067-75.

26. Chiu LQ, Wang W. A case of unusual Gram-negative bacilli septic arthritis in an immunocompetent patient. Singapore Med J 2013;54:e164-8. 
27. Celenligil H, Eratalay K, Kansu E, Ebersole JL. Periodontal status and serum antibody responses to oral microorganisms in Sjögren's syndrome. J Periodontol 1998;69:571-7.

28. Villa A, Wolff A, Narayana N, Dawes C, Aframian DJ, Lynge Pedersen AM, et al. World Workshop on Oral Medicine VI: A systematic review of medication-induced salivary gland dysfunction. Oral Dis 2015 Nov 25. doi: 10.1111/odi.12402. [Epub ahead of print]

29. Aliko A, Wolff A, Dawes C, Aframian D, Proctor G, Ekström J, et al. World Workshop on Oral Medicine VI: clinical implications of medication-induced salivary gland dysfunction. Oral Surg Oral Med Oral Pathol Oral Radiol 2015;120:185-206. 30. Koller MM, Purushotham KR, Maeda N, Scarpace PJ, Humphreys-Beher MG. Desipramine induced changes in salivary proteins, cultivable oral microbiota and gingival health in aging female NIA Fischer 344 rats. Life Sci 2000;68:445-55.

31. Leung WK, Yau JY, Jin LJ, Chan AW, Chu FC, Tsang CS, et al. Subgingival microbiota of renal transplant recipients. Oral Microbiol Immunol 2003;18:37-44. 32. Saraiva L, Lotufo RF, Pustiglioni AN, Silva HT Jr, Imbronito AV. Evaluation of subgingival bacterial plaque changes and effects on periodontal tissues in patients with renal transplants under immunosuppressive therapy. Oral Surg Oral Med Oral Pathol Oral Radiol Endod 2006;101:457-62

33. Diaz PI, Hong BY, Frias-Lopez J, Dupuy AK, Angeloni M, Abusleme L, et al. Transplantation-associated long-term immunosuppression promotes oral colonization 
by potentially opportunistic pathogens without impacting other members of the salivary bacteriome. Clin Vaccine Immunol 2013;20:920-30.

34. Ames NJ, Sulima P, Ngo T, Barb J, Munson PJ, Paster BJ, et al. A characterization of the oral microbiome in allogeneic stem cell transplant patients. PLoS One 2012;7:e47628.

35. Fricke WF, Maddox C, Song Y, Bromberg JS. Human microbiota characterization in the course of renal transplantation. Am J Transplant 2014;14:416-27.

36. Kamada N, Seo SU, Chen GY, Núñez G. Role of the gut microbiota in immunity and inflammatory disease. Nat Rev Immunol 2013;13:321-35.

37. Scher JU, Sczesnak A, Longman RS, Segata N, Ubeda C, Bielski C, et al. Expansion of intestinal Prevotella copri correlates with enhanced susceptibility to arthritis. Elife 2013;2:e01202.

38. Szymula A, Rosenthal J, Szczerba BM, Bagavant H, Fu SM, Deshmukh US. T cell epitope mimicry between Sjögren's syndrome Antigen A (SSA)/Ro60 and oral, gut, skin and vaginal bacteria. Clin Immunol 2014;152:1-9. 
Figure 1. Microbiota composition at the phylum level. Relative abundances of the 10 dominant bacteria phyla found across the two groups are shown as histograms. A $t$ test was used to detect the difference between two groups. Phylum with different relative abundance is marked with $*(P<0.05)$.

Figure 2. The Venn diagram based on genera shows the extent of overlap of buccal mucosa microbiota between SS and control.

Figure 3. Genera with different prevalence in control (white) and SS (black) subjects $(P<$ $0.05)$.

Figure 4. Genera with different relative abundance in control (O) and SS (ᄉ) subjects $(P<$ $0.05)$.

Figure 5. Biplot of redundancy analysis (RDA) of the microbiota composition which responding to PA therapy in SS patients: the plot of the axis 1 (accounting for $18.1 \%$ variation) and the axis 2 (accounting for $1.1 \%$ variation). Whether suffering from the disease of SS or not and whether taking the treatment of PA or not were used as environmental variables. Only 25 most abundant genera (smaller solid triangulars) were included in the analysis. Inverted larger empty triangular: controls (subjects G1-10); star: SS subjects taking only HC (patients 1, 2, 5, 6, 8, 10); circle: SS subjects taking PA and HC (patients 3, 4, 7, 9). 
Table 1 Demographic and clinical characteristics of SS and control subjects

\begin{tabular}{llll}
\hline Characteristic & SS (n=10) & Control (n=10) & Statistic \\
\hline Median Age (years) & 49.5 & 49.5 & $P>0.05$ \\
No. of teeth & $24 \pm 5$ & $25 \pm 4$ & $P>0.05$ \\
Stimulated secretion rate (ml/min) & $0.46 \pm 0.17$ & $1.93 \pm 0.48$ & $P<0.01$ \\
Unstimulated secretion rate (ml/min) & $0.03 \pm 0.02$ & $0.33 \pm 0.11$ & $P<0.01$ \\
Ocular staining score & $6.80 \pm 2.41$ & --- & \\
Focus score & $2.90 \pm 1.66$ & --- & \\
Subjects with positive SS-A & 6 & --- & \\
Subjects with positive SS-B & 3 & -- & \\
\hline
\end{tabular}




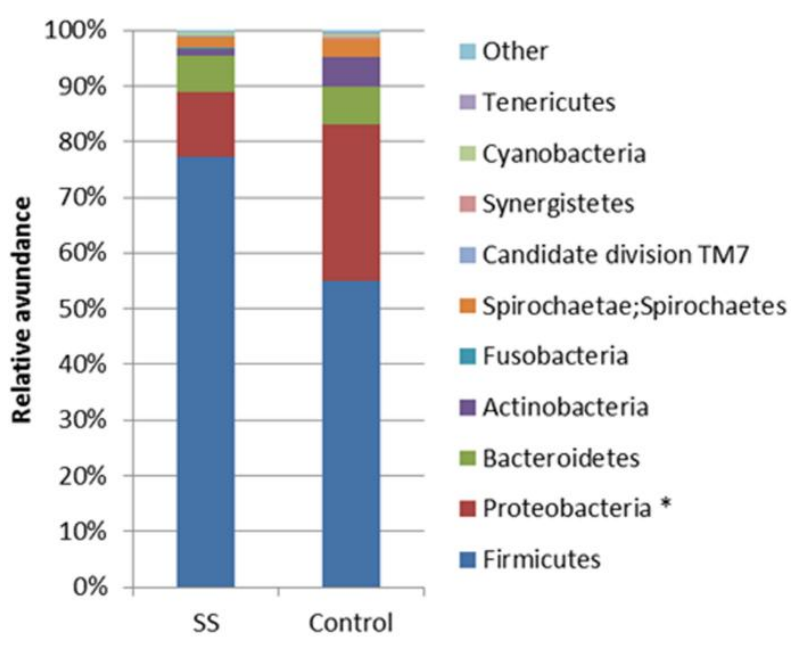




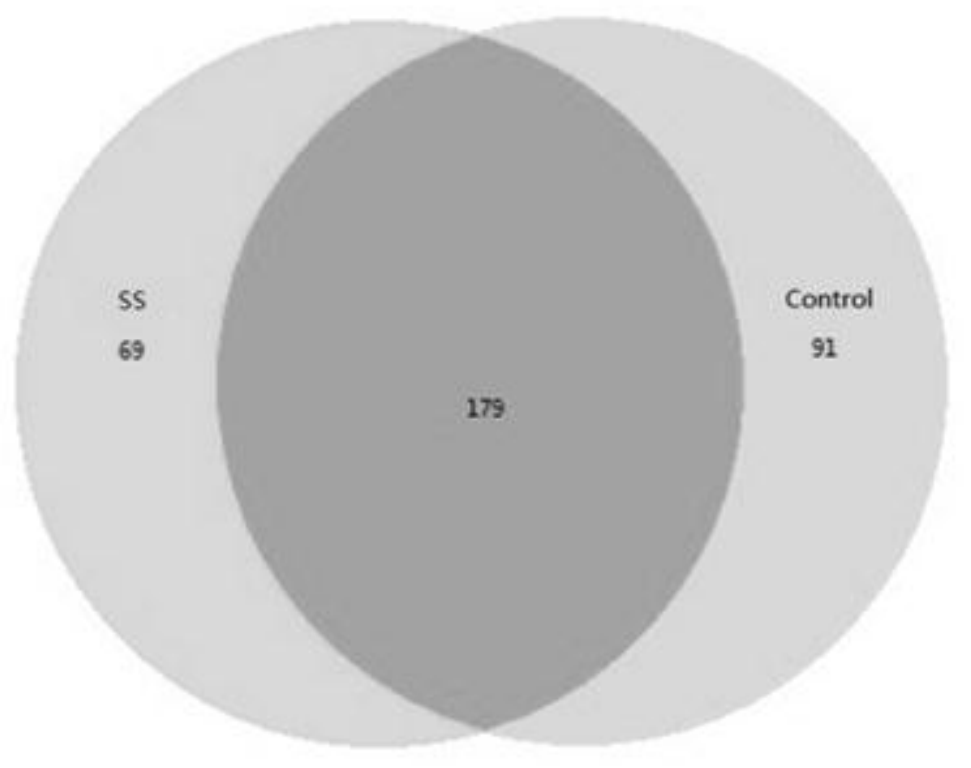




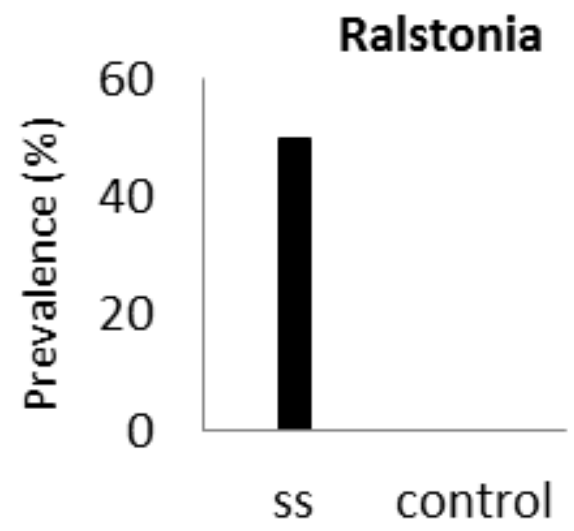




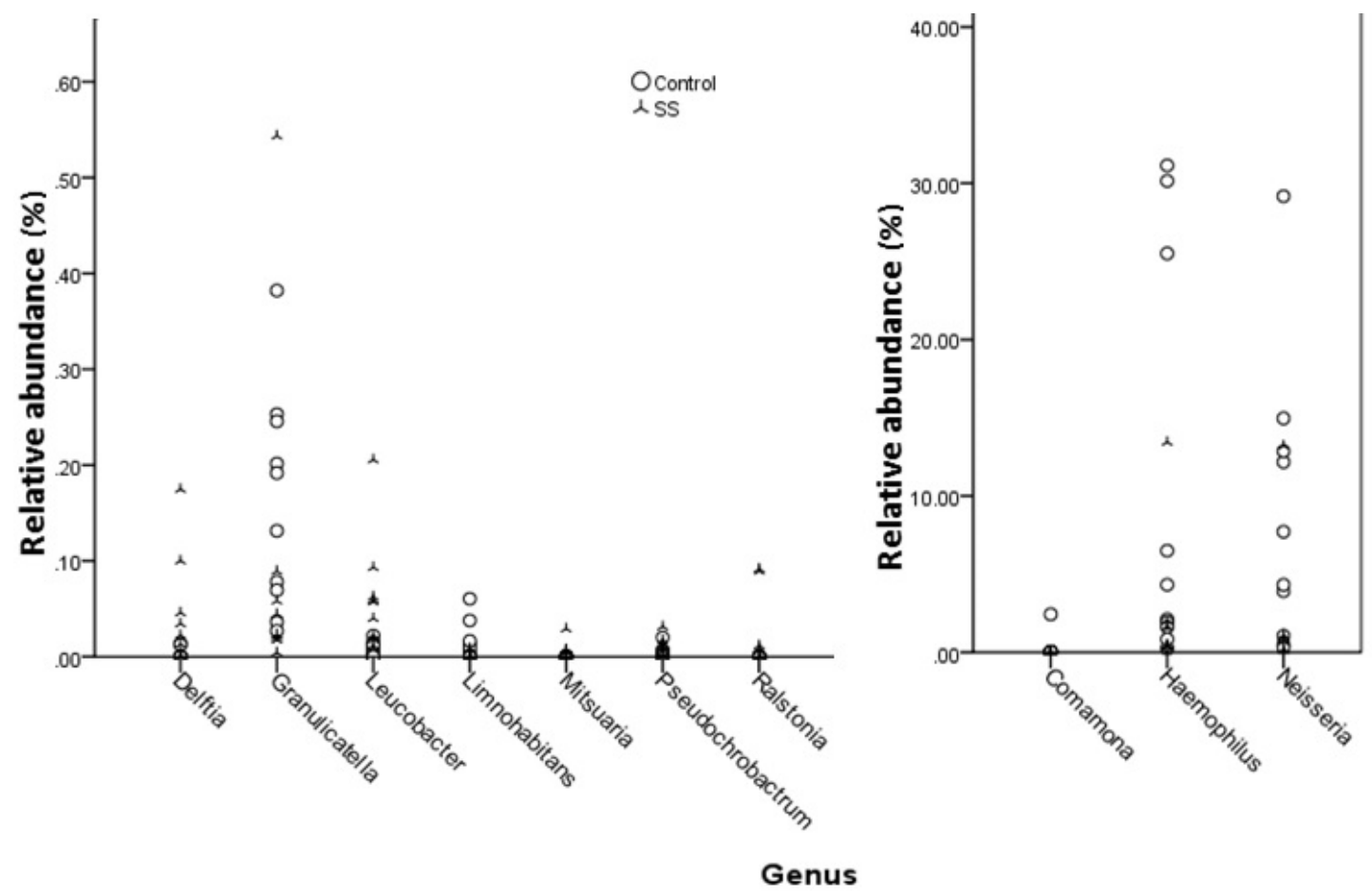




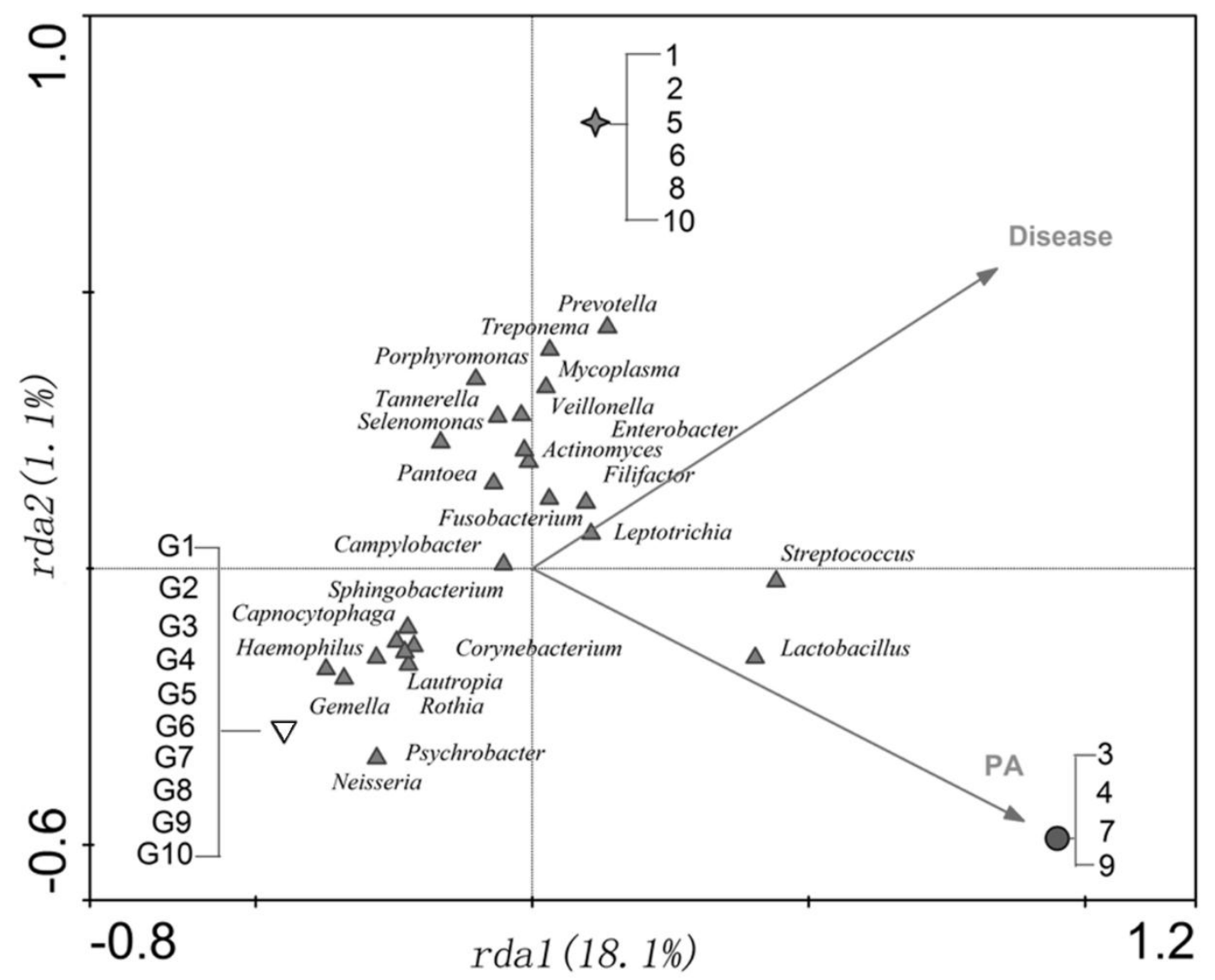

